S. YAMAGUCHI AND G. CHŪMAN

KODAI MATH. J.

6 (1983), $1-13$

\title{
CRITICAL RIEMANNIAN METRICS ON SASAKIAN MANIFOLDS
}

\author{
By SeIIChi Yamaguchi ANd Gorō ChūMan
}

1. Introduction. Let $g$ be a Riemannian metric which is defined on a compact orientable differentiable manifold $M$ of dimension $n$ and makes its volume $V_{g}$ equal to 1 , that is, $\int_{M} d V_{g}=1$, where $d V_{g}$ is the volume element of $M$ measured by $g$. We denote the set of all such metrics by $\mathfrak{M}$. When $g$ is fixed we have a Riemannian manifold $(M, g)$. Let us take a covering $\{U\}$ of $M$ by coordinate neighborhoods and denote the local coordinates in $U$ by $\left\{x^{a}\right\}$, where $a, b, c, \cdots$ run over the range $\{1,2,3, \cdots, n\}$. In each $U, g$ is expressed by its components $g_{a b}$. We adopt summation convention so that the contravariant components $g^{a b}$ of $g$ satisfy $g_{a c} g^{b c}=\delta_{a}^{b}$. By $R_{a b c}{ }^{d}, R_{a b}$ and $R$ we denote the components of the Riemannian curvature tensor, the Ricci tensor and the scalar curvature of $(M, g)$, respectively. Now let us consider the integral

$$
F_{M}[g]=\int_{M} f(R) d V_{g},
$$

where $f(R)$ is a scalar field on $M$ determined by $g$ as the contraction of a tensor product of the curvature tensor. This integral defines a mapping $F: \mathfrak{M} \rightarrow R$. A critical point of $F$ is denoted by $g_{F}$ and is called a critical Riemannian metric with respect to the field $f(R)$ or the integral $F_{M}[g]$. The following four kinds of critical Riemannian metrics have been studied by M. Berger [1] and Y. Mutō $[5,6,7,8,9]$ :

$$
\begin{array}{ll}
A_{M}[g]=\int_{M} R d V_{g}, & B_{M}[g]=\int_{M} R^{2} d V_{g}, \\
C_{M}[g]=\int_{M} R_{a b} R^{a b} d V_{g}, & D_{M}[g]=\int_{M} R_{a b c d} R^{a b c d} d V_{g} .
\end{array}
$$

The equations of the critical Riemannian metric are written as follows:

$$
A_{a b}=C_{A} g_{a b}, \quad B_{a b}=C_{B} g_{a b}, \quad C_{a b}=C_{C} g_{a b}, \quad D_{a b}=C_{D} g_{a b},
$$

where $C_{A}, C_{B}, C_{C}$ and $C_{D}$ are undetermined constants and $A_{a b}, B_{a b}, C_{a b}$ and $D_{a b}$ are given by

Received February 16, 1981 


$$
\begin{aligned}
\text { (1.2A) } \quad A_{a b}= & -R_{a b}+\frac{1}{2} R g_{a b}, \\
\text { (1.2B) } \quad B_{a b}= & 2 \nabla_{a} \nabla_{b} R-2 \nabla_{r} \nabla^{r} R g_{a b}-2 R R_{a b}+\frac{1}{2} R^{2} g_{a b}, \\
(1.2 \mathrm{C}) \quad C_{a b}= & \nabla_{a} \nabla_{b} R-\nabla_{r} \nabla^{r} R_{a b}-\frac{1}{2} \nabla_{r} \nabla^{r} R g_{a b}-2 R_{a s r b} R^{s r}+\frac{1}{2} R_{s r} R^{s r} g_{a b}, \\
(1.2 \mathrm{D}) \quad D_{a b}= & 2 \nabla_{a} \nabla_{b} R-4 \nabla_{r} \nabla^{r} R_{a b}+4 R_{a r} R_{b}{ }^{r}-4 R_{a s r b} R^{s r} \\
& -2 R_{a t s r} R_{b}{ }^{t s r}+\frac{1}{2} R_{t s r q} R^{t s r q} g_{a b},
\end{aligned}
$$

where $\nabla$ means covariant differentiation with respect to the connection induced by $g$. It $1 \mathrm{~s}$ well known that a critical point $g$ of $A_{M}[g]$ in $\mathfrak{M}$ is an Einstein metric.

Although critical Riemannian metrics were first defined on a compact manifold, it is to generalize the definition when $M$ is not compact. The resulting equations are the same as the foregoing ones and (1.1) and (1.2) are valid.

The purpose of this paper is to study the results obtained by Y. Mutō [9] more deeply. $\$ 2$ is recalled the definition of a Sasakian manifold. In $\$ 3$ a Sasakian manifold is studied when $g$ turns out to be a critical Riemannian metric and moreover we consider critical Riemannian metrics in a Sasakian manifold with vanishing $C$-Bochner curvature tensor in $\$ 4$. In $\S 5$ we investigate some conditions to be a critical Riemannian metric $g_{C}$ or $g_{D}$. $\$ 6$ is devoted to the study of a Sasakian submersion $\Pi:(\tilde{M}, \tilde{g}, \tilde{\eta}) \rightarrow\left(B,{ }^{B} g\right)$ where $\tilde{g}$ and ${ }^{B} g$ become critical Riemannian metrics simultaneously.

\section{Sasakian manifold.}

Let $\left(M, g, r_{\jmath}\right)$ be an $n(n \geqq 3)$-dimensional Sasakian manifold (connected and $C^{\infty}$ ) with metric tensor $g$. Then there exist a Killing 1-form $\eta$ satisfying

$$
\begin{aligned}
& \phi_{a^{\prime}}=\nabla_{a} \eta_{b}, \quad \phi_{a b}=-\phi_{b a}, \quad \eta^{a}=g^{a r} \eta_{r}, \quad \eta_{a} \eta^{a}=1, \\
& \phi_{a}^{r} \phi_{r}^{b}=-\delta_{a}^{b}+\eta_{a} \eta^{b}, \quad \nabla_{a} \phi_{b c}=\eta_{b} g_{a c}-\eta_{c} g_{a b} .
\end{aligned}
$$

On a Sasakian manifold $(M, g, \eta)$, the following identities are well known:

$$
\begin{aligned}
& R_{a b c}{ }^{r} \eta_{r}=\eta_{a} g_{b c}-\eta_{b} g_{a c}, \quad R_{a}{ }^{r} \eta_{r}=(n-1) \eta_{a}, \\
& R_{a b s r} \phi_{c}{ }^{s} \phi_{d}{ }^{r}-R_{a b c d}=g_{a c} g_{b d}-g_{b c} g_{a d}-\phi_{a c} \phi_{b d}+\phi_{b c} \phi_{a d}, \\
& R_{s a r b} \phi^{s r}=-S_{a b}+(n-1) \phi_{a b},
\end{aligned}
$$

where we put $S_{a b}=\phi_{a}{ }^{r} R_{r b}$.

We introduce the tensor field $U_{a b c}{ }^{d}$ and $G_{a b}$ in a Sasakian manifold $(M, g, \eta)$, defined by 


$$
\begin{aligned}
U_{a b c}{ }^{d}= & R_{a b c}{ }^{d}-\frac{H+3}{4}\left(g_{b c} \delta_{a}{ }^{d}-g_{a c} \delta_{b}{ }^{d}\right) \\
& -\frac{H-1}{4}\left(g_{a c} \eta_{b} \eta^{d}+\eta_{a} \eta_{c} \delta_{b}{ }^{d}-g_{b c} \eta_{a} \eta^{d}-\eta_{b} \eta_{c} \delta_{a}{ }^{d}\right. \\
& \left.+\phi_{b c} \phi_{a}{ }^{d}-\phi_{a c} \phi_{b}{ }^{d}-2 \phi_{a b} \phi_{c}{ }^{d}\right), \\
G_{a b}= & R_{a b}-\frac{R-n+1}{n-1} g_{a b}-\frac{n(n-1)-R}{n-1} \eta_{a} \eta_{b},
\end{aligned}
$$

where we put $H=\frac{4 R-(n-1)(3 n-1)}{(n-1)(n+1)}$.

A Sasakian manifold $(M, g, \eta)$ is called a space of constant $\phi$-holomorphic sectional curvature $H$ or a locally $C$-Fubinian manifold if its $U_{a b c}{ }^{d}$ vanishes identically. A Sasakian manifold $(M, g, \eta)$ is called an $\eta$-Einstein manifold or a $C$-Einstein manifold if its $G_{a b}$ vanishes identically. In an $\eta$-Einstein manifold ( $n>3$ ), the scalar curvature $R$ is necessarily constant. A 3-dimensional $\gamma$-Einstein manifold means that $R$ is constant.

The equations (2.5) and (2.6) yield the followings:

$$
\begin{aligned}
|G|^{2}=G_{a b} G^{a b}=\left|R_{1}\right|^{2}-\frac{R^{2}}{n-1}+2 R-n(n-1), & \begin{aligned}
|U|^{2}=U_{a b c d} U^{a b c d}= & |R|^{2}+\frac{2}{(n-1)(n+1)}\left\{-4 R^{2}+2(n-1)(3 n-1) R\right. \\
& \left.-n(n-1)^{2}(3 n-1)\right\},
\end{aligned}
\end{aligned}
$$

where we put $|R|^{2}=R_{a b c d} R^{a b c d},\left|R_{1}\right|^{2}=R_{a b} R^{a b}$.

Next we introduce the $C$-Bochner curvature tensor $B_{a b c}{ }^{d}$ in an $n$-dimensional Sasakian manifold given by

$$
\begin{aligned}
B_{a b c d}= & R_{a b c d}+\frac{1}{n+3}-\left(R_{a c} g_{b d}-R_{b c} g_{a d}+g_{a c} R_{b d}-g_{b c} R_{a d}\right. \\
& +S_{a c} \phi_{b d}-S_{b c} \phi_{a d}+\phi_{a c} S_{b d}-\phi_{b c} S_{a d}+2 S_{a b} \phi_{c d}+2 \phi_{a b} S_{c d} \\
& \left.-R_{a c} \eta_{b} \eta_{a}+R_{b c} \eta_{a} \eta_{d}-\eta_{a} \eta_{c} R_{b d}+\eta_{b} \eta_{c} R_{a d}\right) \\
& +\frac{R+(n-1)(n+2)}{(n+1)(n+3)}\left(-\phi_{a c} \phi_{b d}+\phi_{b c} \phi_{a d}-2 \phi_{a b} \phi_{c d}\right) \\
& +\frac{R-3 n-5}{(n+1)(n+3)}\left(-g_{a c} g_{b d}+g_{b c} g_{a d}\right) \\
& +\frac{R+n-1}{(n+1)(n+3)}\left(g_{a c} \eta_{b} \eta_{d}+\eta_{a} \eta_{c} g_{b d}-g_{b c} \eta_{a} \eta_{d}-\eta_{b} \eta_{c} g_{a d}\right)
\end{aligned}
$$

which is constructed from the Bochner curvature tensor in a Kählerian manifold by fibering of Boothby-Wang [3]. 
By straight forward computations the following identities are obtained:

$$
\begin{aligned}
& B_{a b c d}=-B_{b a c d}, \quad B_{a b c d}=B_{c d a b}, \quad B_{a b c}{ }^{d}+B_{b c a}{ }^{d}+B_{c a b}{ }^{d}=0, \quad B_{a b c}{ }^{a}=0, \\
& B_{a b c}{ }^{d} \eta_{d}=0, \quad \phi_{a}^{r} B_{r b c}{ }^{d}=\phi_{b}{ }^{r} B_{r a c}{ }^{d}, \quad \phi^{a b} B_{a b c}{ }^{d}=0, \quad g^{d r} B_{a b c r}=B_{a b c}{ }^{d} .
\end{aligned}
$$

In the rest of the present section, we assume that a Sasakian manifold $(M, g, \eta)$ has the vanishing $C$-Bochner curvature tensor with a constant scalar curvature $R$. Then we have following identity [3].

$$
\nabla_{d} R_{a b}=(n-1)\left(\eta_{a} \phi_{d b}+\eta_{b} \phi_{d a}\right)-\left(\eta_{a} S_{d b}+\eta_{b} S_{d a}\right) .
$$

Operating $\nabla_{e}$ to (2.10) and using the Ricci identity, we have

$$
R_{a s r b} R^{s r}=R_{a r} R_{b}^{r}-R_{a b}+\{R-n(n-1)\}_{\eta_{a}} \eta_{b}+(n-1) g_{a b} .
$$

Also, applying $\nabla^{d}$ to $(2.10)$, we get

$$
\nabla_{r} \nabla^{r} R_{a b}=-2 R_{a b}+2(n-1) g_{a b}+2\left\{R-n(n-1) \eta_{a} \eta_{b} .\right.
$$

Contracting the vanishing $C$-Bochner curvature tensor with $R^{b c}$ and $R_{e}^{b c d}$, we find respectively

$$
\begin{aligned}
& (n+1)(n-1) R_{a r} R_{b}^{r}=(n-1)(R-3 n-5) R_{a b} \\
& \quad+\left\{(n+1)\left|R_{1}\right|^{2}-R^{2}+4(n+1) R-(n-1)\left(n^{2}+3 n+4\right)\right\} g_{a b} \\
& \quad+\left\{-(n+1)\left|R_{1}\right|^{2}+R^{2}-\left(n^{2}+2 n+5\right) R+n(n-1)\left(n^{2}+3 n+4\right)\right\} \eta_{a} \eta_{b}, \\
& (n+1)(n+3) R_{a t s r} R_{e}^{t s r}=16(n+1) R_{e r} R_{a}{ }^{r}-4\left(3 n^{2}-2 n-9+2 R\right) R_{e a} \\
& \quad+2\left\{(n-1)^{2}(3 n+7)-8 R\right\} g_{e a}+8(n+1)\{R-n(n-1)\} \eta_{e} \eta_{a},
\end{aligned}
$$

where we used (2.11). These will be needed later.

\section{Sasakian manifolds with critical Riemannian metrics.}

Let $(M, g, \eta)$ be an $n$-dimensional Sasakian manifold. If the Riemannian metric $g$ is a critical Riemannian metric $g_{B}, g_{C}$ or $g_{D}$, then the undetermined constants $C_{B}, C_{C}$ and $C_{D}$ given by (1.1) are determined as follows [9]:

$$
\begin{aligned}
& C_{B}=\frac{1}{2} R^{2}-2(n-1) R, \\
& C_{C}=\frac{1}{2}\left|R_{1}\right|^{2}-4 R+2(n-1)(n+1), \\
& C_{D}=\frac{1}{2}|R|^{2}-12 R+4(n-1)(3 n-1) .
\end{aligned}
$$

Moreover Y. Mutō [9] has proved 
THEOREM A. If $g$ is a critical Riemannan metrac $g_{A}, g_{B}, g_{C}$ or $g_{D}$ in a Sasakian manifold $(M, g, \eta)$, then scalar curvature is constant.

We first consider the case when $g$ is a critical Riemannian metric $g_{B}$ in a Sasakian manifold $(M, g, \eta)$. By the aid of (1.1), (1.2B) and Theorem A, we can easily see that $(M, g, \eta)$ is an Einstein manifold. Conversely, if $(M, g, \eta)$ is an Einstein manifold, then we can see from (1.2B) that $B_{a b}=C_{B} g_{a b}$. Thus we have

THEOREM 3.1. In a Sasakıan manifold $(M, g, \eta)$, in order that $g$ be a critıcal Riemannian metric $g_{B}$, it is necessary and sufficient that $(M, g, \eta)$ be an Einstern manifold.

Second, let us discuss the case when $g$ is a critical Riemannian metric $g_{C}$ in a Sasakian manifold $(M, g, \eta)$. It follows from Theorem $\mathrm{A},(1.1)$ and $(1.2 \mathrm{C})$ that

$$
C_{C} g_{a b}=-\nabla_{r} \nabla^{r} R_{a b}-2 R_{a s r b} R^{s r}+\frac{1}{2}\left|R_{1}\right|^{2} g_{a b} \text {. }
$$

Transvecting the above equation with $g^{a b}$, we have

$$
C_{C}=\frac{n-4}{2 n}\left|R_{1}\right|^{2}
$$

which and (3.1C) yield

$$
\left|R_{1}\right|^{2}=2 n R-n(n-1)(n+1) .
$$

If we take account of (2.7), then we get

$$
(n-1)|G|^{2}=-R^{2}+2\left(n^{2}-1\right) R-n(n-1)^{2}(n+2) \geqq 0,
$$

that is,

$$
n(n-1) \leqq R \leqq(n-1)(n+2) .
$$

Now, let us examine the following two special cases of (3.2):

(i) the scalar curvature $R$ equals to $n(n-1)$,

(ii) the scalar curvature $R$ equals to $(n-1)(n+2)$.

It is evident that $(M, g, \eta)$ is an Einstein manifold or an $\eta$-Einstein manifold satisfying $R_{a b}=(n+1) g_{a b}-2 \eta_{a} \eta_{b}$ respectively, if the case (i) or (ii) holds.

Thus we have

THEOREM 3.2. If $g$ is a critıcal Riemannan metruc $g_{C}$ in an n-dimensional Sasakıan manifold $(M, g, \eta)$, then the scalar curvature $R$ is pinched with

$$
n(n-1) \leqq R \leqq(n-1)(n+2) \text {. }
$$

Especially, of the scalar curvature $R$ equals to $n(n-1)$ or $(n-1)(n+2)$, then the manifold is Einstern satisfying $R_{a b}=(n-1) g_{a b}$ or $\eta$-Einstein satısfying $R_{a b}=$ $(n+1) g_{a b}-2 \eta_{a} \eta_{b}$ respectively. 
In the rest of this section, we study that $g$ is a critical Riemannian metric $g_{D}$ in a Sasakian manifold $(M, g, \eta)$. From Theorem A, (1.1) and (1.2D) it is clear that

$$
C_{D} g_{a b}=-4 \nabla_{r} \nabla^{r} R_{a b}+4 R_{a r} R_{b}^{r}-4 R_{a s r b} R^{s r}-2 R_{a t s r} R_{b}{ }^{t s r}+\frac{1}{2}|R|^{2} g_{a b},
$$

from which by contraction this with $g^{a b}$ we have

$$
C_{D}=\frac{n-4}{2 n}|R|^{2}
$$

which implies

$$
|R|^{2}=6 n R-2 n(n-1)(3 n-1),
$$

because of (3.1D). This together with (2.8) gives

$$
\left(n^{2}-1\right)|U|^{2}=-8 R^{2}+2(n-1)\left(3 n^{2}+9 n-2\right) R-2 n(n-1)^{2}(n+2)(3 n-1) \geqq 0,
$$

that is,

$$
n(n-1) \leqq R \leqq \frac{1}{4}(n-1)(n+2)(3 n-1) .
$$

If the equalities hold respectively, then we can prove that $(M, g, \eta)$ is of constant curvature 1 or of constant $\phi$-holomorphic sectional curvature $H=3 n-1$.

Therefore we get

ThEOREM 3.3. If $g$ is a critical Riemannian metruc $g_{D}$ in an n-dimensional Sasakıan manfold, then the scalar curvature $R$ is pinched with

$$
n(n-1) \leqq R \leqq \frac{1}{4}(n-1)(n+2)(3 n-1) .
$$

Especially, if the scalar curvature $R$ equals to $n(n-1)$ or $\frac{1}{4}(n-1)(n+2)(3 n-1)$, then the manufold is of constant curvature 1 or of constant $\phi$-holomorphic sectional curvature $H=3 n-1$ respectively.

\section{Critical Riemannian metrics in a Sasakian manifold with vanishing $C$-Bochner curvature tensor.}

If $g$ is a critical Riemannian metric $g_{A}$ or $g_{B}$ in a Sasakian manifold with vanishing $C$-Bochner curvature tensor, then by virtue of Theorem 3.1 we can easily verify that it is of constant curvature 1 .

Next suppose that $g$ is a critical Riemannian metric $g_{C}$ in a Sasakian manifold with vanishing $C$-Bochner curvature tensor, then from Theorem $A$, (1.1), $(1.2 \mathrm{C}),(2.11)$ and $(2.12)$ we find

$$
2(n+1)(n-1) C_{C} g_{a b}=4(n-1)\{(5 n+7)-R\} R_{a b}
$$




$$
\begin{aligned}
& +\left\{(n+1)(n-5)\left|R_{1}\right|^{2}+4 R^{2}-16(n+1) R-4(n-1)\left(n^{2}-3 n-6\right)\right\} g_{a b} \\
& +\left\{4(n+1)\left|R_{1}\right|^{2}-4 R^{2}-4\left(n^{2}-2 n-7\right) R+4 n(n-1)\left(n^{2}-3 n-6\right)\right\} \eta_{a} \eta_{b} .
\end{aligned}
$$

Differentiating covariantly both side of (4.1) and using (2.10), we get

$$
\begin{aligned}
& (n-1)(5 n+7-R)\left(\eta_{a} S_{d b}+\eta_{b} S_{d a}\right)=\left\{(n+1)\left|R_{1}\right|^{2}-R^{2}-2(n+1)(n-3) R\right. \\
& \left.+(n-1)\left(n^{3}+2 n^{2}-4 n-7\right)\right\}\left(\eta_{a} \phi_{d b}+\eta_{b} \phi_{d a}\right) .
\end{aligned}
$$

If we contract this with $\eta^{b} \phi_{c}{ }^{d}$ and make use of (1.1), then we obtain

$$
\begin{aligned}
& (n-1)\{R-(5 n+7)\} R_{a c} \\
& =\left\{-(n+1)\left|R_{1}\right|^{2}+R^{2}+2(n+1)(n-3) R-(n-1)\left(n^{3}+2 n^{2}-4 n-7\right)\right\} g_{a c} \\
& \quad+\left\{(n+1)\left|R_{1}\right|^{2}-R^{2}-\left(n^{2}-2 n-7\right) R+n(n-1)\left(n^{2}-3 n-6\right)\right\} \eta_{a} \eta_{c},
\end{aligned}
$$

from which, by contraction

$$
\left|R_{1}\right|^{2}=2 n R-n(n-1)(n+1) .
$$

Substituting (4.3) into (4.2), we have

$$
\{R-(5 n+7)\}\left[(n-1) R_{a b}-\{R-(n-1)\} g_{a b}+\{R-n(n-1)\} \eta_{a} \eta_{b}\right]=0,
$$

from which $R=5 n+7$ or $R_{a b}=\frac{R-(n-1)}{n-1} g_{a b}+\frac{n(n-1)-R}{n-1} \eta_{a} \eta_{b}$. If the scalar curvature $R$ equals to $5 n+7$, then we can prove by Theorem 3.2 that $n$ is necessarily 7 and the manifold is of constant curvature 1. It follows from (2.7) and (4.2) that the scalar curvature $R$ equals to $n(n-1)$ or $(n-1)(n+2)$ if $R \neq 5 n+7$. Consequently Theorem 3.2 actually shows that $(M, g, \eta)$ is of constant curvature 1 or of constant $\phi$-holomorphic sectional curvature $H=(n+9) /(n+1)$, respectively.

Thus we obtain

THEOREM 4.1. If $g$ is a critical Rremannian metric $g_{C}$ in an n-dimensional Sasakian manfold $(M, g, \eta)$ with vanishing $C$-Bochner curvature tensor, then the manifold is of constant curvature 1 or of constant $\phi$-holomorphic sectional curvature $H=(n+9) /(n+1)$.

Finally we assume that $g$ is a critical Riemannian metric $g_{D}$ in a Sasakian manifold with vanishing $C$-Bochner curvature tensor. From Theorem A, (1.1), (1.2D), (2.11), (2.12) and (2.4) we find

$$
\begin{aligned}
& 2(n-1)(n+1)(n+3) C_{D} g_{a b}=8(n-1)\left(9 n^{2}+32 n+31-4 R\right) R_{a b} \\
& \quad+\left\{16(n+1)(n-5)\left|R_{1}\right|^{2}-8(n-9) R^{2}-4\left(3 n^{3}-3 n^{2}+37 n+91\right) R\right. \\
& \left.\quad+2(n-1)\left(3 n^{4}-27 n^{3}-19 n^{2}+163 n+136\right)\right\} g_{a b} \\
& \quad+\left\{64(n+1)\left|R_{1}\right|^{2}-64 R^{2}-8\left(3 n^{3}+5 n^{2}-19 n-53\right) R\right.
\end{aligned}
$$




$$
\left.+8 n(n-1)\left(3 n^{3}+5 n^{2}-27 n-45\right)\right\} \eta_{a} \eta_{b} \text {. }
$$

Differentiating (4.4) covariantly and making use of (2.10), we get

$$
\begin{aligned}
& (n-1)\left(9 n^{2}+32 n+31-4 R\right)\left(\eta_{a} S_{d b}+\eta_{b} S_{d a}\right) \\
& =\left\{8(n+1)\left|R_{1}\right|^{2}-8 R^{2}-\left(3 n^{3}+9 n^{2}-27 n-49\right) R\right. \\
& \left.\quad+(n-1)\left(3 n^{4}+14 n^{3}-4 n^{2}-46 n-31\right)\right\}\left(\eta_{a} \phi_{d b}+\eta_{b} \phi_{d a}\right),
\end{aligned}
$$

from which we have by contraction of this with $\eta^{b} \phi_{e}{ }^{d}$

$$
\begin{aligned}
(n-1) & \left\{4 R-\left(9 n^{2}+32 n+31\right)\right\} R_{a e} \\
= & \left\{-8(n+1)\left|R_{1}\right|^{2}+\left(3 n^{3}+9 n^{2}-27 n-49\right) R+8 R^{2}\right. \\
& \left.-(n-1)\left(3 n^{4}+14 n^{3}-4 n^{2}-46 n-31\right)\right\} g_{a e} \\
& +\left\{8(n+1)\left|R_{1}\right|^{2}-8 R^{2}-\left(3 n^{3}+5 n^{2}-19 n-53\right) R\right. \\
& \left.+n(n-1)\left(3 n^{3}+5 n^{2}-27 n-45\right)\right\} \eta_{a} \eta_{e},
\end{aligned}
$$

which implies

$$
8(n+1)\left|R_{1}\right|^{2}=4 R^{2}+\left(3 n^{3}+18 n^{2}+9 n-22\right) R-n(n-1)\left(3 n^{3}+14 n^{2}+5 n-14\right) .
$$

Comparing this with (4.5), we have

$$
\left\{4 R-\left(9 n^{2}+32 n+31\right)\right\}\left[(n-1) R_{a b}-\{R-(n-1)\} g_{a b}+\{R-n(n-1)\} \eta_{a} \eta_{b}\right]=0 .
$$

First let us discuss the case of $4 R \neq 9 n^{2}+32 n+31$. Then we can easily verify that the manifold is $\eta$-Einstein with $R=n(n-1)$ or $(n-1)(n+2)(3 n-1) / 4$. Consequently it follows by virtue of Theorem 3.3 that $(M, g, \eta)$ is of constant curvature 1 or of constant $\phi$-holomorphic sectional curvature $H=3 n-1$. The case of $4 R=9 n^{2}+32 n+31$ implies that $n \geqq 7$ by Theorem 3.3.

Gathering these, we have

THEOREM 4.2. Let $g$ be a critical Riemannian metric $g_{D}$ in an n-dimensional Sasakian manifold $(M, g, \eta)$ with vanishing $C$-Bochner curvature tensor. Then

(i) for $4 R \neq 9 n^{2}+32 n+31$, the manfold is of constant curvature 1 or of constant $\phi$-holomorphac sectional curvature $H=3 n-1$,

(ii) for $4 R=9 n^{2}+32 n+31$, the scalar curvature $R$ satısfies the inequality

$$
n(n-1) \leqq R \leqq \frac{1}{4}(n-1)(n+2)(3 n-1), \quad(n \geqq 7) .
$$

\section{Conditions to be critical Riemannian metrics.}

In this section we shall investigate some conditions that $g$ is a critical Riemannian metric $g_{C}$ or $g_{D}$ in a Sasakian manifold $(M, g, \eta)$. 
Let $(M, g, \eta)$ be an $\eta$-Einstein manifold. Then we have

$$
(n-1) R_{a b}=\{R-(n-1)\} g_{a b}+\{n(n-1)-R\} \eta_{a} \eta_{b},
$$

from which

$$
(n-1)\left|R_{1}\right|^{2}=R^{2}-2(n-1) R+n(n-1)^{2} .
$$

Substituting (5.1) into (2.12), we can see

$$
(n-1) \nabla_{r} \nabla^{r} R_{a b}=2\{n(n-1)-R\}\left(g_{a b}-n \eta_{a} \eta_{b}\right) .
$$

Substituting (5.1) and (5.2) into (2.13), we have

$$
(n-1)^{2} R_{a r} R_{b}^{r}=\{R-(n-1)\}^{2} g_{a b}+\{n(n-1)-R\}\{R+(n-1)(n-2)\} \eta_{a} \eta_{b} .
$$

From (5.1), (5.4) and (2.11), we can get

$$
\begin{aligned}
(n-1)^{2} R_{a s r b} R^{s r}= & \left\{R^{2}-3(n-1) R+(n-1)^{2}(n+1)\right\} g_{a b} \\
& -\{R-n(n-1)\}\{R-2(n-1)\} \eta_{a} \eta_{b} .
\end{aligned}
$$

Consequently we get

$$
\begin{aligned}
2(n-1)^{2} C_{a b}= & \left\{(n-5) R^{2}-2(n-1)(n-9) R+(n-1)^{2}\left(n^{2}-9 n-4\right)\right\} g_{a b} \\
& +4\{R-n(n-1)\}\{R-(n-1)(n+2)\} \eta_{a} \eta_{b}
\end{aligned}
$$

by virtue of (1.2C), (5.2), (5.3) and (5.5). Therefore we find

THEOREM 5.1. In an n-dimensional $\eta$-Einstern manifold, if the scalar curvature $R$ equals to $n(n-1)$ or $(n-1)(n+2)$, then $g$ is a critical Riemannian metruc $g_{C}$.

As for a critical Riemannian metric $g_{D}$ we shall prove

THEOREM 5.2. In an n-dimensional Sasakian manifold $(M, g, \eta)$ of constant $\phi$-holomorphic sectional curvature $H$, if $H$ equals to 1 or $3 n-1$, then $g$ is a critical Riemannian metruc $g_{D}$.

Proof. It is well known that the $C$-Bochner curvature tensor coincides with $U_{a b c}{ }^{d}$ if and only if $(M, g, \eta)$ is an $\eta$-Einstein manifold. Thus, $(M, g, \eta)$ is considered as an $\eta$-Einstein manifold with vanishing $C$-Bochner curvature tensor. Thus we have the following equation by the aid of (2.8):

$$
|R|^{2}=\frac{2}{(n-1)(n+1)}\left\{4 R^{2}-2(n-1)(3 n-1) R+n(n-1)^{2}(3 n-1)\right\} .
$$

Substituting (5.1) and (5.4) into (2.14), we have

$$
\begin{aligned}
(n+1)(n-1)^{2} R_{a t s r} R_{b}{ }^{t s r}= & 2\left\{4 R^{2}-2(n-1)(3 n-1) R+(n-1)^{3}(3 n+1)\right\} g_{a b} \\
& -4\{R-n(n-1)\}\left\{2 R-(n-1)^{2}\right\} \eta_{a} \eta_{b} .
\end{aligned}
$$


Compering with (1.2D), (5.3), (5.4), (5.5), (5.6) and (5.7), we find

$$
\begin{aligned}
(n-1)^{2}(n+1) D_{a b}= & \left\{4(n-5) R^{2}-2(n-1)\left(3 n^{2}-22 n-1\right) R\right. \\
& \left.+(n-1)^{2}\left(3 n^{3}-28 n^{2}-3 n+4\right)\right\} g_{a b} \\
& +4\{R-n(n-1)\}\{4 R-(n-1)(n+2)(3 n-1)\} \eta_{a} \eta_{b},
\end{aligned}
$$

which shows that the assertion of the theorem is true.

\section{Sasakian submersion.}

We consider Riemannian submersions $\Pi:(\tilde{M}, \tilde{g}) \rightarrow\left(B,{ }^{B} g\right)$ such that fibers $F$ are complete and connected and imbedded in $(\tilde{M}, \tilde{g})$ regularly as totally geodesic submanifolds. The Riemannian metrics on the total manifold $\tilde{M}$, the base manifold $B$ and the fiber $F$ are denoted respectively by $\tilde{g},{ }^{B} g$ and ${ }^{F} g$. Let $(\tilde{M}, \tilde{g}, \tilde{\eta})$ be a Sasakian manifold where $\operatorname{dim} \tilde{M}=\tilde{n}$. Let the indices $a, b, c, d, e, \cdots$ run over the range $\{1,2, \cdots, \tilde{n}\}$ and the indices $h, \imath, \jmath, k, \cdots$ the range $\{1,2, \cdots, n\}$ where $\tilde{n}=n+1$. A Sasakian manifold $(\tilde{M}, \tilde{g}, \tilde{\eta})$ admits a Riemannian submersion where the unit Killing vector $\tilde{\eta}$ is a vertical vector and the fibers are geodesics tangent to $\tilde{\eta}$. Such a Riemannian submersion is called a Sasakian submersion.

Let us recall some relations with respect to a Sasakian submersion $\Pi:(\tilde{M}, \tilde{g}, \tilde{\eta})$ $\rightarrow\left(B,{ }^{B} g\right)$. (For details see [9], [14]). For the Riemannian metric ${ }^{B} g$ on the base manifold $B$ we have

$$
{ }^{B} g_{\jmath \imath}=\tilde{g}_{\jmath i}-\tilde{\eta}_{j} \tilde{\eta}_{2}, \quad{ }^{B} g^{j i}=\tilde{g}^{j i} .
$$

Relation between the curvature tensor $\tilde{R}_{a b c}{ }^{d}$ of $(\tilde{M}, \tilde{g}, \tilde{\eta})$ and the curvature tensor ${ }^{B} R_{k j 2}{ }^{h}$ of $\left(B,{ }^{B} g\right)$ has be given as follows:

$$
\left(R^{11}\right)_{k \jmath \imath}{ }^{h}={ }^{B} R_{k \jmath i}{ }^{h}-F_{j i} F_{k}{ }^{h}+F_{k i} F_{\jmath}{ }^{h}+2 F_{k j} F_{\imath}{ }^{h}, \quad\left(F_{j i}=F_{\jmath}{ }^{r B} g_{r \imath}\right),
$$

where $R^{H}$ denotes the horizontal part of the curvature tensor $\tilde{R}$ of $(\tilde{M}, \tilde{g}, \tilde{\eta})$ and $F_{j}{ }^{2}$ represents a complex structure $J$ such that $\left(B,{ }^{B} g, J\right)$ is a Kählerian structure on $B$. For the Ricci tensor and the scalar curvature we have

$$
\begin{aligned}
& \tilde{R}_{j \imath}={ }^{B} R_{j i}-2^{B} g_{j \imath}+(\tilde{n}-1) \tilde{\eta}_{\jmath} \tilde{y}_{\imath \imath}, \\
& \tilde{R}={ }^{B} R-(\tilde{n}-1) .
\end{aligned}
$$

In a Riemannian manifold $(M, g)$, since the tensors $B, C$ and $D$ are given by (1.2), corresponding tensors of a Sasakian manifold $(\tilde{M}, \tilde{g}, \tilde{\eta})$ will be denoted by $\widetilde{B}, \widetilde{C}$ and $\widetilde{D}$ and their components by $\widetilde{B}_{a b}, \widetilde{C}_{a b}$ and $\widetilde{D}_{a b}$, while these of the base manifold $\left(B,{ }^{B} g\right)$ by ${ }^{B} B,{ }^{B} C$ and ${ }^{B} D$ and their components by ${ }^{B} B_{\imath \jmath},{ }^{B} C_{\imath \jmath}$ and ${ }^{B} D_{\imath \jmath}$. If the scalar curvature $\tilde{R}$ of $(\tilde{M}, \tilde{g}, \tilde{\eta})$ is constant, the following identities are obtained by Y. Muto [9]:

$$
\tilde{B}_{\imath \jmath}={ }^{B} B_{\imath \jmath}+2(\tilde{n}-1)^{B} R_{\imath \jmath}+\left\{-(\tilde{n}-5)^{B} R-4(\tilde{n}-1)+\frac{1}{2}(\tilde{n}-1)^{2}\right\}^{B} g_{\imath \jmath}+\tilde{C}_{B} \tilde{\eta}_{\imath} \tilde{\eta}_{\jmath},
$$




$$
\begin{aligned}
& \tilde{C}_{\imath \jmath}={ }^{B} C_{\imath j}+12^{B} R_{\imath j}+\left\{-2^{B} R+\frac{1}{2}(\tilde{n}+3)(\tilde{n}-9)\right\}^{B} g_{\imath j}+\tilde{C}_{C} \tilde{\eta}_{\imath} \tilde{\eta}_{\jmath}, \\
& \tilde{D}_{\imath \jmath}={ }^{B} D_{\imath j}+36^{B} R_{\imath j}+\left\{-6^{B} R+(3 \tilde{n}+2)(\tilde{n}-9)\right\}^{B} g_{\imath j}+\tilde{C}_{D} \tilde{\eta}_{\imath} \tilde{\eta}_{\jmath},
\end{aligned}
$$

where we put

$$
\begin{aligned}
& \tilde{C}_{B}=\frac{1}{2}\left({ }^{B} R\right)^{2}-3(\tilde{n}-1)^{B} R+\frac{5}{2}(\tilde{n}-1)^{2}, \\
& \widetilde{C}_{C}=\frac{1}{2}{ }^{B} R_{\imath \jmath}{ }^{B} R^{\imath \jmath}-6^{B} R+\frac{5}{2}(\tilde{n}-1)(\tilde{n}+3), \\
& \widetilde{C}_{D}=\frac{1}{2}{ }^{B} R_{\imath \jmath k l}{ }^{B} R^{\imath j k l}-18^{B} R+5(3 \tilde{n}+2)(\tilde{n}-1) .
\end{aligned}
$$

Y. Muto [9] has proved the following theorems:

Theorem B. Let $I I:(\tilde{M}, \tilde{g}, \tilde{\eta}) \rightarrow\left(B,{ }^{B} g\right)$ be a Sasakzan submersion. If $\tilde{g}$ and ${ }^{B} g$ are critical Riemannian metrics $g_{B}$ on $\tilde{M}$ and $B$ respectively, then the scalar curvature $\widetilde{R}$ is constant and $\left(B,{ }^{B} g\right)$ is an Einstern manfold satisfying

$$
\left({ }^{B} R\right)^{2}-(\tilde{n}-1)(\tilde{n}+2)^{B} R+(\tilde{n}-1)^{2}(\tilde{n}+1)=0 .
$$

Conversely consider the case where $\left(B,{ }^{B} g\right)$ is an Einstem manfold where ${ }^{B} R=$ $\tilde{n}^{2}-1$ or $\tilde{n}-1$. If one of $\tilde{g}$ and ${ }^{B} g$ is a critical Riemannan metruc $g_{B}$, then the other is also a critical Riemannian metrics $g_{B}$.

Theorem C. Let $\Pi:(\tilde{M}, \tilde{g}, \tilde{\eta}) \rightarrow\left(B,{ }^{B} g\right)$ be a Sasakian submersion. If $\tilde{g}$ and ${ }^{B} g$ are critucal Riemannian metrics $g_{C}$ on $\tilde{M}$ and $B$ respectively, then the scalar curvature $\tilde{R}$ is constant and $\left(B,{ }^{B} g\right)$ is an Einstein manifold satisfying $(*)$. Conversely, consider the case where $\left(B,{ }^{B} g\right)$ is an Einstein manifold satisfying (*). If one of $\tilde{g}$ and ${ }^{B} g$ is a critical Riemannian metruc $g_{C}$, then the other is also a critical Riemannian metric $g_{C}$. Where $\left(^{*}\right)$ is as follows.

$$
\left({ }^{B} R\right)^{2}-2(\tilde{n}-1)(\tilde{n}+2)^{B} R+(\tilde{n}+1)(\tilde{n}+3)(\tilde{n}-1)^{2}=0 .
$$

TheOREM D. Let $\Pi:(\tilde{M}, \tilde{g}, \tilde{\eta}) \rightarrow\left(B,{ }^{B} g\right)$ be a Sasakian submersion. If $\tilde{g}$ and ${ }^{B} g$ are critical Riemannan metrics $g_{D}$ on $\tilde{M}$ and $B$ respectively, then the scalar curvature $\widetilde{R}$ is constant and $\left(B,{ }^{B} g\right)$ is an Einstein manfold satısfynng (**). Conversely, let us consider the case where $\left(B,{ }^{B} g\right)$ is an Einstem manifold satisfying (**). If one of $\tilde{g}$ and ${ }^{B} g$ is a critical Riemannian metric $g_{D}$, then the other is also a critical Riemannian metric $g_{D}$. Where $(* *)$ is as follows

$$
{ }^{B} R_{\imath j k l}{ }^{B} R^{\imath j k l}=6(\tilde{n}+2)^{B} R-2(3 \tilde{n}+2)\left(\tilde{n}^{2}-1\right) .
$$

We shall now show the following:

THEOREM 6.1. Let $I:(\tilde{M}, \tilde{g}, \tilde{\eta}) \rightarrow\left(B,{ }^{B} g\right)$ be a Sasakzan submersion. In order 
that $\tilde{g}$ be a critical Riemannian metruc $g_{B}$ in a Sasakian manifold $(\tilde{M}, \tilde{g}, \tilde{\eta})$, it is necessary and sufficient that $\left(B,{ }^{B} g\right)$ be an Einstein manifold satisfying

$$
{ }^{B} R_{a b}=(\tilde{n}+1)^{B} g_{a b},
$$

and ${ }^{B} g$ be a critical Riemannian metric $g_{B}$.

Proof. If we assume that $\tilde{g}$ is a critical Riemannian metric $g_{B}$ on $\tilde{M}$, then, it follows from Theorem 3.1 that $(\tilde{M}, \tilde{g}, \tilde{\eta})$ is an Einstein manifold satisfying

$$
\tilde{R}_{a b}=(\tilde{n}-1) \tilde{g}_{a b} \text {. }
$$

In view of (6.1), this means that ${ }^{B} R_{\imath \jmath}=(\tilde{n}+1)^{B} g_{\imath \jmath}$, namely, the base manifold $\left(B,{ }^{B} g\right)$ is an Einstein one with ${ }^{B} R=(\tilde{n}-1)(\tilde{n}+1)$. Furthermore from (6.5B) we have ${ }^{B} B_{\imath \jmath}={ }^{B} C_{B}{ }^{B} g_{\imath \jmath}$, because of $\tilde{g}$ is a critical Riemannian metric $g_{B}$. The converse is trivial by Theorem B. These complete the proof.

Secondly, let us prove

THEOREM 6.2. Let $\Pi:(\tilde{M}, \tilde{g}, \tilde{\eta}) \rightarrow\left(B,{ }^{B} g\right)$ be a Sasakian submersion and $\tilde{g}$ be a critical Riemannian metric $g_{C}$ in a Sasakian manifold $(\tilde{M}, \tilde{g}, \tilde{\eta})$. Then the scalar curvature ${ }^{B} R$ in $\left(B,{ }^{B} g\right)$ is pinched with

$$
(\tilde{n}-1)(\tilde{n}+1) \leqq{ }^{B} R \leqq(\tilde{n}-1)(\tilde{n}+3) .
$$

Especially, if the scalar curvature ${ }^{B} R$ equals to $(\tilde{n}-1)(\tilde{n}+1)$ or $(\tilde{n}-1)(\tilde{n}+3)$, then the manifold is an Einstern one satisfying

$$
{ }^{B} R_{a b}=(\tilde{n}+1)^{B} g_{a b} \text { or }{ }^{B} R_{a b}=(\tilde{n}+3)^{B} g_{a b}
$$

respectiveiy, and ${ }^{B} g$ is a critıcal Riemannian metric $g_{C}$ in $\left(B,{ }^{B} g\right)$.

Proof. Taking account of the fact that $\tilde{g}$ is a critical Riemannian metric $g_{C}$, in view of Theorem 3.2 and (6.4) it is easy to see that the scalar curvature ${ }^{B} R$ in $\left(B,{ }^{B} g\right)$ is pinched with (6.7). Recalling that if the scalar curvature ${ }^{B} R$ of $\left(B,{ }^{B} g\right)$ is $\tilde{n}^{2}-1$ or $(\tilde{n}-1)(\tilde{n}+3)$, then the scalar curvature $\tilde{R}$ of $(\tilde{M}, \tilde{g}, \tilde{\eta})$ is $\tilde{n}(\tilde{n}-1)$ or $(\tilde{n}-1)(\tilde{n}+2)$ respectively, we can easily see by virtue of Theorem 3.2 that $(\tilde{M}, \tilde{g}, \tilde{\eta})$ is Einstein or $\eta$-Einstein satisfying $\tilde{R}_{a b}=(\tilde{n}+1) \tilde{g}_{a b}-2 \tilde{\eta}_{a} \tilde{\eta}_{b}$ respectively. Therefore it is evident by (6.3) that $\left(B,{ }^{B} g\right)$ is an Einstein manifold satisfying (6.8). Consequently, regarding to $\widetilde{C}_{a b}=\widetilde{C}_{C} \tilde{g}_{a b},(6.1)$ and the mentions above, we have ${ }^{B} C_{\imath \jmath}={ }^{B} C_{C}{ }^{B} g_{\imath \jmath}$, which means that ${ }^{B} g$ is a critical Riemannian metric $g_{C}$. These facts show that the assertion of the theorem is true.

Finally we shall prove the following

Theorem 6.3. Let $\Pi:(\tilde{M}, \tilde{g}, \tilde{\eta}) \rightarrow\left(B,{ }^{B} g\right)$ be a Sasakıan submersion and $\tilde{g}$ be a critical Riemannian metric $g_{D}$ in a Sasakian manifold $(\tilde{M}, \tilde{g}, \tilde{\eta})$. Then the scalar curvature ${ }^{B} R$ in $\left(B,{ }^{B} g\right)$ is pinched with

$$
(\tilde{n}-1)(\tilde{n}+1) \leqq B ~ R \leqq \frac{1}{4}(\tilde{n}-1)(\tilde{n}+1)(3 \tilde{n}+2) .
$$


Especially, of the scalar curvature ${ }^{B} R$ equal to $\tilde{n}^{2}-1$ or $(\tilde{n}-1)(\tilde{n}+1)(3 \tilde{n}+2) / 4$, then $\left(B,{ }^{B} g\right)$ is of constant holomorphic sectional curvature $H=4$ or $H=3 \tilde{n}+2$ respectively, and ${ }^{B} g$ is a critucal Riemannian metruc $g_{D}$.

Proof. As $\tilde{g}$ is a critical Riemannian metric $g_{D}$, it follows from (6.4) and Theorem 3.3 that $(6.9)$ holds. If ${ }^{B} R$ is $\tilde{n}^{2}-1$ or $(\tilde{n}-1)(\tilde{n}+1)(3 \tilde{n}+2) / 4$, we have $\tilde{R}=\tilde{n}(\tilde{n}-1)$ or $(\tilde{n}-1)(\tilde{n}+2)(3 \tilde{n}-1) / 4$ respectively. So we can see from Theorem 3.3 that $(\tilde{M}, \tilde{g}, \tilde{\eta})$ is of constant curvature 1 or of constant $\phi$-holomorphic sectional curvature $\tilde{H}=3 \tilde{n}-1$. Making use of $(6.2)$, we have that $\left(B,{ }^{B} g\right)$ is of constant holomorphic sectional curvature $H=4$ or $3 \tilde{n}+2$ respectively. These facts prove that ${ }^{B} g$ is a critical Riemannian metric $g_{D}$. These complete the proof.

The authors wish to express their sincere thanks to Professor Y. Mutō who gave kind encouragements.

\section{REFERENCES}

[1] M. Berger, Quelques formules de variation pour une structure riemannienne, Ann. sc1. Ecole Norm. Sup., $4^{\mathrm{e}}$ serie, 3 (1970), 285-294.

[2] S. IshinARA AND M. Konishi, Differential geometry of fibred spaces, Publications of the study group of geometry, Tokyo, 8 (1973).

[3] M. Matsumoto and G. Chüman, On the $C$-Bochner curvature tensor, TRU Math., 5 (1969), 21-30.

[4] Y. Mutō, On some properties of a fibred Riemannian manifold, Scı. Rep. Yokohama Nat. Univ., Sect. I, No. 1 (1952), 1-14.

[5] Y. Mutō, On Einsteın metrics, J. Diff. Geometry, 9 (1974), 521-530.

[6] Y. Mutō, Curvature and critical Riemannian metrics, J. Math. Soc. Japan, 26 (1974), 686-697.

[7] Y. Mutō, Critıcal Riemannıan metrıcs, Tensor N. S., 29 (1975), 125-133.

[8] Y. Mutō, Critıcal Riemannıan metrıcs on product manıfolds, Kōdaı Math. Sem. Rep., 26 (1975), 409-423.

[9] Y. Mutō, Riemannıan submersions and critıcal Riemannian metrıcs, J. Math. Soc. Japan, 29 (1977), 493-511.

[10] T. Nagano, On fibred Riemannian manifolds, Scı. Papers College Gen. Ed. Univ. Tokyo, 10 (1960), 17-27.

[11] B. O'Neill, The fundamental equations of a submersion, Michigan Math. J., 13 (1966), 459-469.

[12] S. SASAKI, Almost contact manıfolds I, Lecture note, Tôhoku Unıv., (1965).

[13] K. Yano ANd S. Ishihara, Differential geometry of fibred spaces, Kōdal Math. Sem. Rep., 19 (1967), 257-288.

[14] K. Yano and S. Ishihara, Fibred spaces with invariant Riemannian metrics, Kōdai Math. Sem. Rep., 19 (1967), 317-360.

Department of Mathematics

Science University of Tokyo

TOKYO JAPAN 162

AND

Department of Mathematics

FACULTy of Education

Gifu University

GIFU, JAPAN 502 